\title{
Frontières
}

\section{Entrevue avec Chloé Sainte-Marie}

\section{Aimée Verret}

Volume 22, numéro 1-2, automne-printemps 2009-2010

Résilience et deuil

URI : https://id.erudit.org/iderudit/045037ar

DOI : https://doi.org/10.7202/045037ar

Aller au sommaire du numéro

Éditeur(s)

Université du Québec à Montréal

ISSN

1180-3479 (imprimé)

1916-0976 (numérique)

Découvrir la revue

Citer ce document

Verret, A. (2009). Entrevue avec Chloé Sainte-Marie. Frontières, 22(1-2),

123-124. https://doi.org/10.7202/045037ar d'utilisation que vous pouvez consulter en ligne.

https://apropos.erudit.org/fr/usagers/politique-dutilisation/ 


\section{Entrevue avec CHLOÉ SAINTE-MARIE}

\section{Aimée Verret \\ Collaboratrice de Chloé Sainte-Marie}

Plusieurs connaissent Chloé SainteMarie pour son parcours artistique: ses rôles au cinéma, ses albums où elle chante avec fougue des poètes québécois et amérindiens. Pourtant, au cours des dernières années, elle a endossé son plus récent rôle auprès de Gilles Carle, son conjoint: celui d'aidante naturelle. Le cinéaste, qui a vécu plus de 17 ans avec la maladie de Parkinson, a eu besoin de soins spécialisés constants. Dans cette entrevue réalisée avant le décès de Gilles Carle le 28 novembre 2009, Chloé SainteMarie partage avec nous son expérience et ses souvenirs. Elle nous parle aussi de ses démarches pour venir en aide à d'autres familles touchées par la maladie.

A. V.: Selon vous, qu'est-ce que la résilience? En quoi cela s'applique-t-il à votre situation?

C. S.-M. : La résilience, ça me fait penser à un bon caoutchouc qui résiste! Je me sens comme ça parfois. Gilles vit avec la maladie de Parkinson depuis plus de 17 ans, alors je pense être un véritable spécimen de résilience! (Rire) Mon exemple du caoutchouc reflète un peu cette indestructibilité devant l'épreuve, une résistance aux multiples chocs que la maladie nous assène sans relâche. On vit un stress intense et on n'a d'autre choix que de s'y adapter.

A. V.: Gilles Carle est sûrement lui aussi un modèle de résilience.

C. S.-M.: Oui, plus que moi! C'est véritablement un modèle de force et de courage. Il continue de résister, résister. Il voit mourir son corps, mais son esprit est toujours là, vivant. Il est confiné au silence et a perdu petit à petit toute motricité, toute autonomie. S'il demeure terriblement fort, c'est grâce à son désir de vivre. Dans un des textes qu'il a publiés, il parle de «l'espoir que demain, ça aille mieux». Moi, je lutte avec la force du désespoir. Lui, c'est avec la force de l'espoir!
A. V.: Au cours de votre relation avec Gilles Carle, vous avez dû faire face à nombre de deuils. Pouvez-vous nous parler du principal deuil que vous avez eu à faire, celui que vous avez trouvé le plus difficile?

C. S.-M.: Je pense que Gilles et moi avons un point de vue différent sur cette question. Personnellement, je dirais que j'ai vécu mon principal deuil lorsqu'il a perdu la parole. Pour moi, Gilles, c'est l'homme de la parole. Mais pour lui, je pense que le plus gros deuil a vraiment été l'écriture, le dessin. C'était ainsi qu'il s'exprimait. Tout partait de l'écriture, pour lui. Ses scénarios, ses livres, ses pièces... Gilles écrivait tout. Lorsqu'il est tombé malade et qu'il ne pouvait plus dessiner, mon amie Bibitte ${ }^{1}$ m'a raconté qu'un jour, elle l'a vu caresser ses crayons.

A. V.: Lorsque vous regardez en arrière dans votre vie, pouvez-vous nous parler de ce qui, de votre point de vue, vous a aidée à être résiliente? 
C. S.-M. : C'est probablement mon sens de la responsabilité qui a fait que je suis devenue résiliente. J'aurais pu partir à tout moment; Gilles m'a même dit, en 1998: «Va-t'en! Pars!» J'étais libre, mais j'ai fait un choix conscient, celui de rester. Et en même temps, j'ai fait le choix d'être aidante. En 1996, j'avais presque abandonné la partie; nous avions même fait le pacte de mourir ensemble. Nous étions partis plusieurs mois à l'île Verte. Au fond, je pense que cela va au-delà du sens de la responsabilité; j'aurais été incapable d'agir autrement que je l'ai fait.

A. V.: Vous aviez un choix, mais au fond, ce n'en était pas un.

C. S.-M.: Je ne sentais pas que j'avais le choix. J'ai pris cette décision parce que c'était la seule admise à mes yeux. Pour moi, on ne peut pas abandonner quelqu'un qu'on aime. Une mère n'abandonne pas son enfant parce qu'il est malade. En fait, soi-même, on ne sait pas qu'on est résilient; ce sont les autres qui peuvent voir cette force en nous. On n'en a pas conscience.

A. V.: D'après vous, est-ce que chacun d'entre nous porte une telle résilience?

C. S.-M. : Ça ne m'est pas particulier, je l'ai toujours dit. Il y a des milliers d'aidants naturels au Québec. Je reçois chaque jour des témoignages de gens qui soignent leur père, leur mère, leur mari, leur enfant. Je ne suis pas exceptionnelle, je ne fais rien d'extraordinaire. Je fais le geste ordinaire de quelqu'un qui aime. C'est l'amour, ce n'est pas la compassion. S'il n'y avait pas d'amour, je ne le ferais pas.

A. V.: Pourquoi avez-vous choisi de soigner Gilles Carle chez lui?

C. S.-M.: Parce que les mouroirs me tuent! (Rire) J'en ai visité plusieurs et j'ai décidé que ce n'était pas une solution. Je n'allais pas le laisser dépérir dans un mouroir dans une humiliation constante. Le placer dans une résidence n'a jamais été un choix ni même une possibilité pour moi.

A. V.: Que retirez-vous personnellement de votre expérience d'aidante?

C. S.-M. : Ça m'a apporté la conscience de l'essentiel. J'étais très insouciante, indolente. Je vivais dans le paraître, dans l'égocentrisme. Gilles et moi étions dans une autre sphère, loin de la Terre. Nous vivions dans une bulle, dans son imaginaire. Moi, j'étais heureuse comme ça. Être aidante m'a appris l'altruisme, la découverte de l'autre. Avec Gilles, on était un; je n'avais pas l'impression qu'on était deux. C'est aujourd'hui que je réalise à quel point les amis sont importants. J'ai tant besoin de les voir! Avant, je ne connaissais pas ce besoin-là. Gilles me comblait à cent pour cent.
A. V.: Si l'amour et l'aide de vos amis sont pour vous des piliers sur lesquels vous vous appuyez, y a-t-il toutefois quelque chose qui pourrait vaincre cette force et même vous mener au désespoir?

C. S.-M.: La seule chose qui pourrait vaincre cette force serait de perdre la santé à mon tour, car je n'aurais plus la capacité de continuer. C'est une peur qui me hante. Maintenant, je prends soin de moi, mais c'est un nouveau réflexe. J'ai été dans le plus grand trou noir de ma vie à l'été 2007. Il n'y avait plus de solution; je pensais perdre la maison. Je donnais quand même les représentations de mon spectacle Parlemoi. Heureusement car sinon, j'y serais sûrement passée. Je me raccrochais à mon équipe, qui était comme ma famille. Mes amis ont fait office de psychologues pour moi. Ils sont venus, ils m'ont parlé, m'ont convaincue de prendre soin de moi. Puis, j'ai eu l'idée de faire une maison d'accueil, d'abord dans une église; ça a passé par plusieurs stades. Ensuite, j'ai pensé vendre les dessins de Gilles dans un coffret d'art qui s'appelle Variations sur un "t'aime». Mes amis proches, Jacques Lacoursière, Bruno Roy, Charles Binamé, Bibitte, Pierre Dury, m'ont soutenue et le projet s'est mis en branle. J'avais maintenant une raison de me battre; j'ai commencé à vendre le coffret et à ramasser des sous.

A. V.: Vous avez beaucoup lutté pour obtenir de l'assistance pour les soins et de l'argent pour pouvoir continuer. La population en général aux prises avec les mêmes problèmes n'a pas nécessairement les ressources et les moyens de faire ce que vous avez fait. Que pouvez-vous leur dire pour les aider à continuer et à développer leur résilience?

C. S.-M.: Il faut lutter, il faut se battre, tout faire pour être entendus. Chacun a une tribune qui lui est propre et il faut l'utiliser. Mon rêve, ce serait une coalition entre tous les aidants pour que nous descendions dans la rue et que nous manifestions. Mais les aidants n'ont pas d'argent, ils n'ont pas de temps, ils sont épuisés.

A. V.: Selon votre expérience et vos souvenirs, en quoi le rôle d'aidant n'est pas facile?

C. S.-M. : À cause du manque de reconnaissance. Chacun a besoin d'une reconnaissance sociale et financière. Des gens qui travaillent sans salaire, j'en connais personnellement peu. Nous n'avons ni aide ni soutien pour payer des préposés, donc pas de répit. La solitude avec le malade est très difficile. Même si j'étais riche, je fonderais une maison d'accueil pour briser cet isolement, pour permettre aux personnes malades et à leurs familles de se rassembler. Je ne suis plus capable de fournir seule l'énergie et la dédramatisation nécessaires. J'ai besoin d'aide.

A. V.: C'est d'autant plus dur que les personnes malades ne sont pas acceptées dans la société.

C. S.-M.: Oui. C'est un cercle vicieux; les aidants se retrouvent aussi isolés que les malades. D'ailleurs, certaines personnes trouvent que je sors trop avec Gilles. On m'a même dit: «Cache-le. » Voir quelqu'un de malade nous ramène inexorablement à notre propre maladie, notre propre mort.

A. V.: Qu'est-ce qui vous a amenée à créer le Réseau des aidants ${ }^{2}$ ?

C. S.-M. : C'est un lieu de rencontre virtuel pour briser la solitude, cet isolement des aidants dont nous parlons. Un lieu où on peut échanger, se parler.

A. V.: Lorsque la personne malade n'arrive plus à communiquer librement, comme c'est le cas pour Gilles Carle, comment vivre la relation avec elle?

C. S.-M.: Gilles communique avec moi par le regard. Il ne peut plus hocher la tête pour faire signe que oui ou non, mais il peut le dire par moment. Je trouve très difficile de ne pas savoir ce qu'il pense, de ne pas connaître ses besoins. Est-ce qu'il a mal, est-ce que ses préposés le traitent bien? Mais parfois, il se produit encore des choses tout à fait extraordinaires. Par exemple, tout récemment, je lui parlais au téléphone. J'étais à Montréal, et lui à Saint-Paul. J'ai dit: "Est-ce que ça va bien, Gilles? » et il a répondu: «Ça va bien. » J'en avais les larmes aux yeux! Dans son état, dire «ça va bien »! Il faut qu'il le veuille vraiment!

A. V.: Lui parlez-vous moins qu'avant?

C. S.-M. : Je lui parle plus! Je parle toute seule! Et je me réponds! (Rire) C'est ça qui me manque le plus: ses réponses. Gilles, je lui posais toutes les questions du monde, tout, tout, tout. C'était mon professeur pour l'attitude à avoir dans la vie, pour la réflexion, l'écriture, le regard sur l'art. J'ai donc perdu mon conseiller principal, car il est devenu muet. Voilà le pire drame de ma vie. Maintenant, je demande conseil à plusieurs de mes amis chers. Un jour, j'ai dit à Gilles: «Tu vois, ça prend au moins six personnes pour te remplacer! » Il riait!

\section{Notes}

1. Bibitte est le surnom de Joséphine Bacon, réalisatrice, poète, et amie du couple.

2. Voir: <http://www.reseaudesaidants. org/>. Chloé Sainte-Marie a aussi créé en novembre 2009 un organisme à but non lucratif, La Maison Gilles-Carle, pour accueillir dans un environnement chaleureux et baigné de culture de grands malades en perte d'autonomie. Voir également: <http://www.maisongilles carle.org/>. 